\title{
Tail myonecrosis in brown shrimp (Crangon crangon L.) caught in the North Sea
}

\author{
Vermeersch Xavier ${ }^{1}$, Koen Chiers², Annemie Decostere ${ }^{2}$ and Geertrui Vlaemynck ${ }^{1}$ \\ 1 Technology and Food Science Research Unit, Institute for Agricultural and Fisheries Research \\ (ILVO), Brusselsesteenweg 370, 9090 Melle, Belgium \\ E-mail: Xavier.Vermeersch@ilvo.vlaanderen.be \\ 2 Veterinary Pathology, Faculty of Veterinary Medicine, UGent, Salisburylaan 133, 9820 Merelbeke, \\ Belgium
}

Myonecrosis of the tail has been observed in cultured Pacific white shrimp (Litopenaeus vannamei) and is caused by Infectious Myonecrosis Virus (IMNV). A similar condition (White Tail Disease) has been reported in freshwater prawns (Macrobrachium rosenbergii) caused by $M$. rosenbergii nodavirus and Extra Small Virus like particle. Additionally, Vibrio harvyei infections in L. vannamei induced similar lesions. Here we demonstrate myonecrosis of the tail in wild caught European brown shrimp.

During the period 2014 - 2015, several batches of brown shrimp were caught by commercial fishing vessels at two month intervals within the 12 mile zone of the Belgium North Sea coast. Shrimp were transferred to the experimental facility within 2 hours after landing and stocked in well aerated seawater tanks. Within 3 weeks, a minority of shrimp developed a circumferential black delineation at the tail with a whitish discoloration of the muscle tissue in the distal end of the carapace and demonstrated a progressive reduction in movement leading to a localised paralysis of the affected region. Samples were taken for histological and virological examination by means of RT-PCR for IMNV.

Upon histological examination, all shrimp presented a distinct coagulative muscle necrosis. A secondary bacterial invasion of the necrotic muscle was additionally observed in the shrimp who often remained alive up to several weeks after the appearance of the first symptoms.

In the hepatopancreas, inclusions of intranuclear bacilliform virus were consistently encountered albeit in low numbers. RT-PCR for IMNV was negative in all samples.

This is the first report of tail necrosis in brown shrimp caught in the North Sea, resembling infectious myonecrosis. However, the etiology still needs to be elucidated.

Keywords: crangon; brown shrimp; myonecrosis; white tail; paralysis 